\title{
Pulse-Height-Spectrum Distortion in Xenon Gaseous Detectors for Soft X Rays: Experimental Results
}

\author{
F. P. Santos, J.M.F.dos Santos, T. H. V. T. Dias and C.A.N.Conde \\ Physics Department, University of Coimbra, P-3000 Coimbra, Portugal
}

\section{Abstract}

The results of an experimental determination of the pulseheight distortion in soft $x$-ray spectra in gaseous xenon detectors are presented. The study confirms previous Monte Carlo simulation results and emphasizes the importance of the electric field in the drift region in reducing spectral degradation while the effect of a strong magnetic field in the same region is shown to be neglegible.

\section{INTRODUCTION}

Xenon-filled gas proportional scintillation counters (GPSC) play an important role in the detection of $x$ rays in many applications such as $x$-ray astronomy $[1,2]$ and energy dispersive $x$-ray fluorescence analysis (EDXRF) $[3,4]$. GPSCs are structurally simple room temperature detectors with high detection efficiency, large window area, high counting rate capability and good energy resolution (a factor of $\sim 2$ better than proportional counters). These characteristics make GPSCs competitive with other radiation detectors in many applications. For energies below 2-3 keV, GPSC's energy resolution can even be better than cryogenic solid state detectors (HPGe and $\mathrm{Si}(\mathrm{Li})$ ) when sensitive areas of several $\mathrm{cm}^{2}$ are required.

For soft $\mathrm{x}$ rays, however, the pulse-height spectra of a GPSC may exhibit distortions represented by a significant low-energy tail (a departure from the gaussian shape output in the low amplitude region), eventually accompanied by a small shift in the peak position, as first observed by Inoue $e t$ al. with a xenon GPSC [5]. These authors have explained the observed x-ray energy dependence of the distortion effect in terms of a loss of primary electrons during drift and diffusion of the primary electron cloud towards the GPSC scintillation region.

A recent detailed Monte Carlo simulation study of the absorption of $x$ rays in gaseous xenon predicted important spectra distortions which translated into a low-energy tail $[6,7]$, and proved that this tail effect could be associated with the loss of primary electrons to the detector entrance window. Furthermore, the Monte Carlo calculation was able to relate this kind of distortion to low $\mathrm{x}$-ray penetration depth in the gas, and showed that the effect was very sensitive to the electric field in the absorption/drift region. Monte Carlo calculations also predicted detectable shifts in the peak position as well. However, these were clearly ascribable to discontinuities in energy linearity near the absorption edges of the filling xenon gas [7,8], and not to primary electron loss to the window, as claimed in Inoue et al. [5] for similar conditions.

In the present work we have carried out experimental confirmation of the Monte Carlo calculated results on the distortion of pulse-height distributions for soft $x$ rays in the 1 to $4.5 \mathrm{keV}$ energy range. The effect of increasing the electric field in the absorption/drift region, as well as the effect of a strong magnetic field, were also investigated.

\section{EXPERIMENTAL SETUP}

The detector used in this experimental study of the degradation of soft $x$-ray energy spectra was a xenon-filled GPSC operated at 780 torr, shown in fig.1 [4]. The detector's entrance window was a $3.5 \mu \mathrm{m}$ thick, $20-\mathrm{mm}$ diameter, aluminized Mylar film, externally supported by a stainless steel grid. The electric field in the $1-\mathrm{cm}$ scintillation region was maintained at $4.5 \mathrm{Vcm}^{-1}$ torr ${ }^{-1}$ for all measurements.

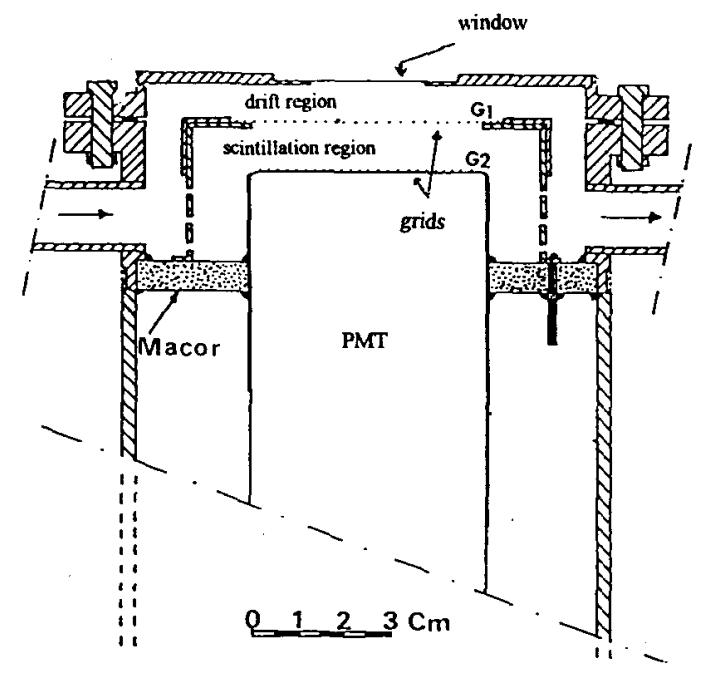

Fig. 1- GPSC schematic.

To study the effect of the electric field, the depth of the absorption/drift region was kept constant at $7 \mathrm{~mm}$. The scintillation light was collected by an EMI-D676 photomultiplier. In this configuration, the energy resolution of the system for a $2-\mathrm{mm}$, collimated $5.9 \mathrm{keV} \mathrm{x}$-ray beam was $8.8 \%$. 
For the study of the effect of an applied magnetic field, a permanent ring magnet $(\mathrm{Nd}-\mathrm{Fe}-\mathrm{B}, 19.0 \times 6.5 \times 10.0 \mathrm{~mm})$ was positioned over the detector entrance window, and the driftregion depth was increased to $40 \mathrm{~mm}$ to reduce the effect of the magnetic field on the PMT. The PMT, in this case, was an EMT-9956QB. Additional magnetic-field reduction was provided by a Conetics cylindrical shield around the PMT. The Conetics shield was also used to support the grid G1. The energy resolution for a 6-mm, collimated $5.9 \mathrm{keV} x$-ray beam was $11.5 \%$ in the absence of the magnetic field, and $12.9 \%$ when it was applied.

The $\mathrm{x}$-ray energies studied, from $\sim 1.2$ to $\sim 4.5 \mathrm{keV}$, were the fluorescence $\mathrm{K}$ lines of $\mathrm{Mg}(1245 \mathrm{eV}), \mathrm{Al}(1487 \mathrm{eV}), \mathrm{Si}$ $(1740 \mathrm{eV}), \mathrm{S}(2307 \mathrm{eV}), \mathrm{Cl}(2622 \mathrm{eV}), \mathrm{Ca}(3690 \mathrm{eV})$ and $\mathrm{Ti}$ $(4508 \mathrm{eV})$ samples.

\section{RESULTS AND DISCUSSION}

For each $\mathrm{x}$-ray energy, a series of increasing electric fields in the drift region was used, in the pressure reduced electric field $\mathrm{E} / \mathrm{p}$ interval 0.1 to $1 \mathrm{Vcm}^{-1}$ torr $^{-1}\left(1 \mathrm{Vcm}^{-1}\right.$ torr $^{-1}$ is the threshold for secondary scintillation $[9,10])$. As an example, the spectra obtained for the highest and the lowest drift electric fields for two different $X$-ray energies $E_{X}$ are shown in figs. 2 and 3 (K lines of $\mathrm{Mg}$ and $\mathrm{Al}$, absorption lenghts $\mathrm{d}_{\mathrm{x}}$ of 296 and $428 \mu \mathrm{m}$ respectively, at 780 torr).

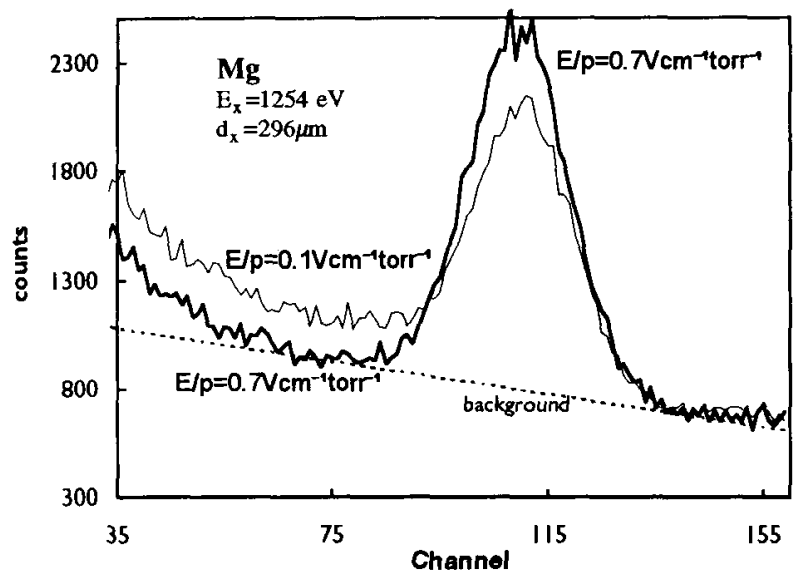

Fig. 2 - $1254 \mathrm{eV} x$-ray spectra for $E / p=0.1$ and $0.7 \mathrm{Vcm}^{-1}$ torr $^{-1}$.

In each figure, an enhanced low-energy tail associated with a decrease in the area under the peak is observed as a function of the applied electric field, the enhancement being greatest for the lowest electric field. This effect can be attributed to the loss of electrons to the entrance window, as established by the Monte Carlo calculations. In addition, no shift in peak position was observed when the electric field was varied, also consistent with the results of the Monte Carlo simulation (see fig. 2 or fig. 3 ).

The likely source for the shift in peak position described in Inoue et al. [5], which was there claimed to be related to electron losses to the window during diffusion, can possibly be attributed to a reduction in secondary scintillation collection related to solid angle effects in the conditions of their experiment.

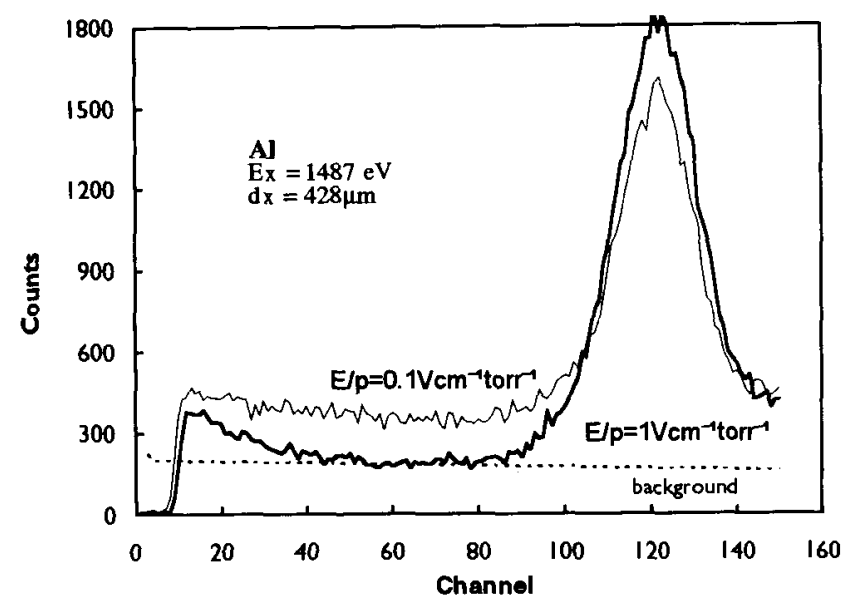

Fig. 3 - $1487 \mathrm{eV} \mathrm{x}$-ray spectra for $\mathrm{E} / \mathrm{p}=0.1$ and $1 \mathrm{Vcm}^{-1}$ torr $^{-1}$

In the $x$-ray energies studied, the area under the lowenergy tail decreases as the $x$-ray energy increases (i.e. as the absorption length increases). For example, for the titanium $\mathrm{K}$ line at $4508 \mathrm{eV}$, the area under the low-energy tail is small even for the lowest value of drift field studied ( $\sim 5 \%$ of the total area)

To characterize the distortion in each spectrum, for both Monte Carlo [6] and experimental studies, a distortion parameter $Q$ was defined as the ratio between the area $\mathrm{T}$ under the tail below the spectrum corresponding to a full electron collection (the reference spectrum $R$ ) and the spectra total area $A$

$Q=\frac{T}{A}$

However, the reference spectrum $R$ cannot be obtained experimentally, as electron losses to the window will always occur for the $x$-ray energies studied and for all reduced drift fields used $\left(<1 \mathrm{Vcm}^{-1}\right.$ torr $^{-1}$ as stated above). Therefore, in this experimental study $Q$ was taken as (see fig.4)

$Q=\frac{\mathrm{T}^{\prime}+\mathrm{T}_{0}}{A}$

where $T^{\prime}$ is the tail area of the current spectrum above the highest $E / p$ spectrum (lowest losses) and $T_{0}$ is the tail area of this spectrum above the hypothetical reference $\mathcal{R}$ As again $\mathrm{T}_{0} / \mathrm{A}$ cannot be deduced from the experimental results, it was assumed that the small distortion it represents is similar, for 
each x-ray energy, to the corresponding value $Q_{0}^{\mathrm{MC}}$ calculated by the Monte Carlo simulation in [6]. The experimentally deduced distortion parameter $Q$ was therefore computed as

$Q=\frac{\mathrm{T}^{\prime}}{A}+\frac{\mathrm{T}_{0}}{A} \sim \frac{\mathrm{T}^{\prime}}{A}+Q_{0}^{\mathrm{MC}}$

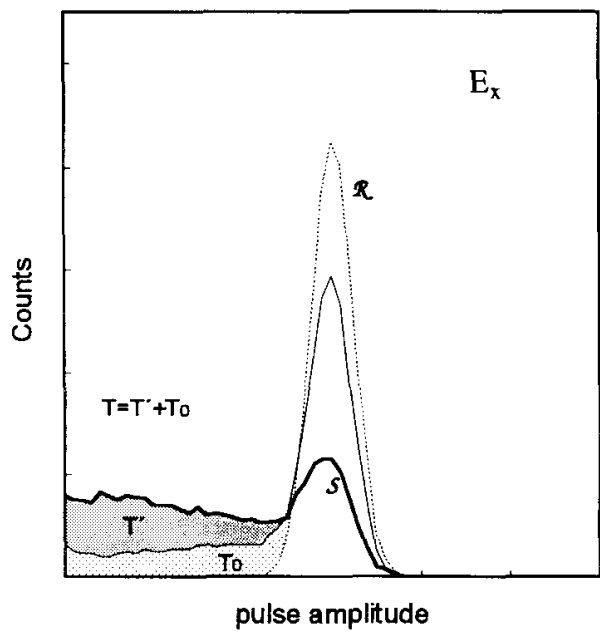

Fig. 4 - Distortion parameter $Q$ for spectrum $S$ is $Q=\left(\mathrm{T}^{\prime}+\mathrm{T}_{0}\right) / A$.

In fig. 5 we compare the distortion parameter $Q$ calculated by Monte Carlo simulation with the experimental values obtained through eq.(3). Fig. 5 shows that the two groups of

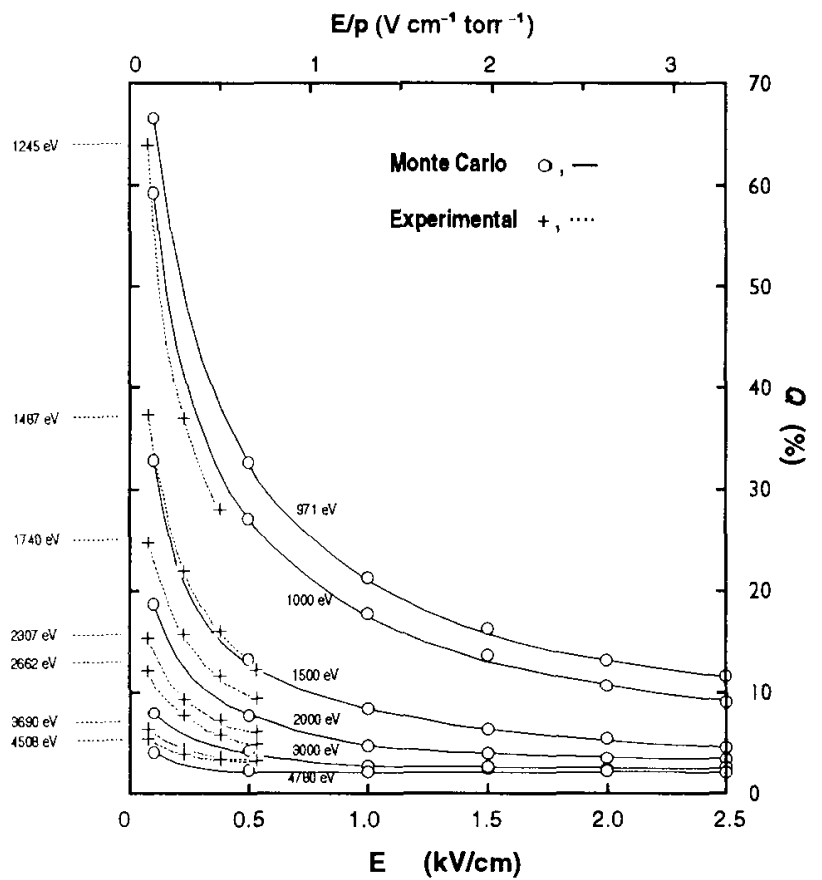

Fig. 5 - Monte Carlo calculated (-O- - ) and experimental $(\cdots+\cdots)$ distortion parameter $Q$ for different x-ray energies absorbed in xenon. results exhibit a very similar behaviour with increasing electric field (note that the behaviour of each experimental curve is not affected by the additive term $Q_{0}^{\mathrm{MC}}$ introduced in eq.(3) which stands only a reference point for each curve).

The $Q$ curves shown in fig. 5 (each curve characterizes an $x$-ray energy $E_{x}$ ), exhibit a systematic decrease with the increase in the drift field $\mathrm{E}$, and it is clear that a judicious choice of the intensity of the electric field applied in the drift region of a conventional GPSC can significantly improve the collection of electrons, and consequently optimize its performance. It should be noted that $\mathrm{E} / \mathrm{p} \sim 1 \mathrm{Vcm}^{-1}$ torr $^{-1}$ is the highest electric field that can be applied in the drift region without jeopardizing the performance of a xenon filled gas proportional scintillation counter, as this $E / p$ value is the threshold for secondary scintillation in xenon. Taking the curve for $E_{x}=1487 \mathrm{eV}$ as an example, it can be seen that for a reduced drift field $\mathrm{E} / \mathrm{p}=0.1 \mathrm{Vcm}^{-1}$ torr $^{-1} Q$ will reach $\sim 35 \%$, while for $\mathrm{E} / \mathrm{p}=0.3 \mathrm{Vcm}^{-1}$ torr $^{-1} Q$ is $\sim 20 \%$, and for $\mathrm{E} / \mathrm{p}=0.7 \mathrm{Vcm}^{-1}$ torr $^{-1} Q \sim 10 \%$.

While distortion is not significant for the higher $\mathrm{x}$-ray energies and for all drift electric fields $\left(E_{x}\right.$ above $\sim 3 \mathrm{keV}$, lower lying curves in fig.5), important electron losses can never be avoided for $x$-ray energies lower than $\sim 1.5 \mathrm{keV}$. In fact, for $\mathrm{E}_{\mathrm{x}}<1.5 \mathrm{keV}, Q$ never reaches a value under $\sim 10 \%$ even for the highest drift field $\left(E / p=1 \mathrm{Vcm}^{-1}\right.$ torr $\left.^{-1}\right)$.

In an attempt to further improve the performance of GPSCs for soft $x$ rays, we have also studied the effect of a strong magnetic field in the absorption region. The effect of the magnetic field on electron trajectories could, in principle, increase the number of electron collisions for those electrons that spiral towards the window. This, in turn, would result in an increased loss in electron energy and a reduction in the probability of capture by the window. For this purpose, a permanent magnet $(\mathrm{Nd}-\mathrm{Fe}-\mathrm{B}$ maximum field intensity $0.2 \mathrm{~T}$ )

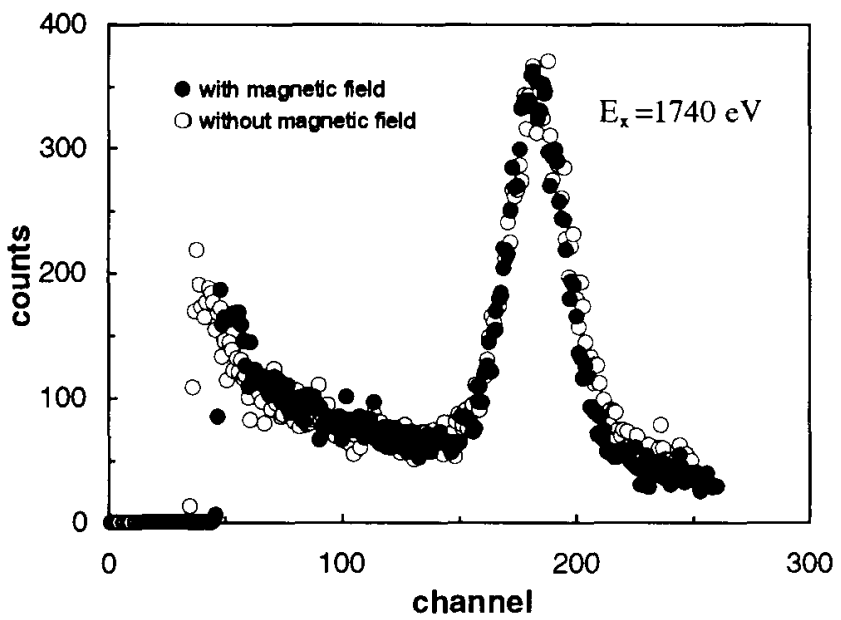

Fig. 6 - Si K line $\left(E_{x}=1740 \mathrm{eV}\right)$ energy spectra obtained with and without the application of a strong magnetic field in the GPSC drift region. 
was placed over the entrance window. Fig. 6 presents the Si K line $(1740 \mathrm{eV})$ spectra obtained with and without the influence of the magnetic field in the GPSC's drift region.

From this example, it is clear that a magnetic field of that intensity has no visible effect in reducing the observed distortion in soft $x$-ray energy spectra. Stronger magnetic fields are expected to be efficient, but the photomultiplier would certainly be affected in the present GPSC's design .

\section{CONCLUSIONS}

Experimental results on the influence of the electric field applied to the drift region of a GPSC in reducing the distortion effects observed in soft $x$-ray energy spectra have been presented in this work. These results were found to be in very good agreement with previous Monte Carlo calculations [6]. The observed distortions in pulse-height distributions are attributed to electron loss to the detector entrance window that result from low-energy $x$ rays that interact with xenon at shallow depths beneath the window. To characterize the distortion effects, a parameter $Q$ has been defined as the tail to total area ratio. The $Q$ results of a systematic study are summarized in fig.5, and allow the choice of the best experimental working conditions for GPSC applications. However, for $\mathrm{E}_{\mathrm{x}}<1.5 \mathrm{keV}$ a value lower than $10 \%$ for $Q$ is never achieved, even for the highest drift fields.

The effect of a weak magnetic field applied to the drift region in minimizing the distortion effect was also experimentally assessed and found to be negligible.

\section{Acknowledgements}

Support is acknowledged from JNICT (Junta Nacional de Investigação Científica e Tecnológica) through project STRDA/P/CEN/422/92.

We thank Dr. R. E. Morgado (LANL, USA) for helpful suggestions.

\section{REFERENCES}

[1] A.Peacock, B.G.Taylor, N.White,T.Courvoisier and G.Manzo, IEEE Trans. Nucl. Sci. NS-32 (1985) 108.

[2] D.F.Anderson, T.T.Hamilton, W.HKu and R. Novick, Nucl. Instrum. Meth. 163 (1969) 125.

[3] D.A. Goganov, A.A.Schultz and B.V.Elkind,. Instrum. Exp. Techn. (URSS) 27 (1984) 478.

[4] J.M.F. dos Santos, A.C.S.S.Bento and C.A.N.Conde, X-Ray Spectrom. 22 (1993)328.

[5] H.Inoue, K.Koyama, M.Matsuoka, T.Ohashi, Y.Tanaka, and H. Tsunemi, Nucl. Instrum. Meth. 1573 (1978) 295.

[6] T.H.V.T.Dias, F.P.Santos, A.D.Stauffer and C.A.N.Conde, Phys. Rev. A46 (1992) 237.

[7] F.P.Santos, T.H.V.T.Dias, A.D.Stauffer and C.A.N.Conde, Nucl. Instrum. Meth. A307 (1991) 347.

[8] T.H.V.T.Dias, F.P.Santos, A.D.Stauffer and C.A.N.Conde, Phys. Rev. A48 (1993) 2887.

[9] F.P.Santos, T.H.V.T.Dias, A.D.Stauffer and C.A.N.Conde, J. Phys. D: Appl. Phys. 27 (1994) 42.

[10] F.Favata, A.Smith, M. Bavdaz,T.Z.Kowalski, Nucl. Instrum. Meth. A294 (1990) 356. 